\title{
Study on Photodynamic Therapy Instrument Based on Photonic Crystals
}

\author{
Li Ping ${ }^{1, a^{*}}$, Zhang Lanlan, Xu Mengli, Fan Ting, Liang Gaofeng \\ ${ }^{1}$ School of Medical Technology and Engineering, Henan University of Science and Technology, \\ Henan Luoyang 471003 \\ E-mail: pingli818@163.com
}

\begin{abstract}
Keywords: photonic crystal, transmission matrix, photodynamic therapy instrument, photodynamic therapy

Abstract. The forefront of photonic crystal theory is applied in this paper to solve such technical difficulties as low color purity, unsatisfactory effect of deep treatment that are commonly seen in photodynamic therapy instruments. By using the transfer matrix method, band gap property of photonic crystals is analyzed through Matlab programming, so as to select appropriate photonic crystal materials and to determine its structural parameters. Research shows that the newly designed photonic crystal structure boasts of good filtering characteristics, allowing only the pass-through of 980nm near infrared light, which has therapeutic effect on liver cancer, gastric cancer and other visceral organ cancers. The photonic crystal filter designed can be used on ordinary photodynamic therapy instrument, and greatly improve its cancer treatment.
\end{abstract}

\section{Introduction}

A Photodynamic therapy (PDT) is widely used for cancer treatment. In PDT, photosensitizers undergo photochemical reactions with light under the influences of oxygen. Research finds that irradiation light with longer wavelength enjoys higher tissue penetrating power, and that the $980 \mathrm{~nm}$ infrared light can be used for the treatment of stomach cancer, liver cancer and other inner organ cancers which call for certain depth of therapy.

At present, the commonly used PDT instruments are RBL-PDT, LED-PDT, ALA-PDT and so on. Most of these instruments use semiconductor laser transmitter as the radiation source. The color purity of the radiation light, however, is quite low. And the photochemical effect of the photosensitizers is constrained because of the light's poor penetrating power at specific depth and low energy utilization ratio. Thus in clinical treatment, longer radiation time is needed, and focal curative effect at specific depth is not satisfactory. It is thus imperative to find a better way to solve the problem of low color purity in PDT.

Photonic crystals(PhCs), introduced by E. Yablonovitch and S. John in 1987, has drawn wide attention ever since the $21^{\text {st }}$ century as a new type of photonic behavior control material. One of the most basic properties of PhCs is band gap, for which it only allows the transmission of light of specific frequencies. Based on this property, it can be used to filter lights.

Through simulation experiment, appropriate PhCs structure and configuration are determined, and high-quality filter of narrow band gap is produced allowing only the transmission of the 980nm near infrared light. When used on laser transmitter of photodynamic therapy instrument, they help to improve the color purity and utilization ratio of the irradiation light, and thus greatly enhance the treatment effect for stomach cancer, liver cancer and other inner organ cancers.

\section{Computation model for PhCs}

Transmission matrix is used here.

To the $\mathrm{N}$ layer dielectric media, field vectors are ${ }^{E_{N}}, H_{N}$ on the left side, $E_{N+1}, H_{N+1}$ on the right side.

$$
\left[\begin{array}{l}
E_{N} \\
H_{N}
\end{array}\right]=M_{N}\left[\begin{array}{l}
E_{N+1} \\
H_{N+1}
\end{array}\right]
$$


Where $M_{N}$ is the transmission matrix of the $N$ layer dielectric media. Thus the transmission matrix for the $N-1$ layer dielectric media is

$$
\left[\begin{array}{c}
E_{N-1} \\
H_{N-1}
\end{array}\right]=M_{N-1}\left[\begin{array}{l}
E_{N} \\
H_{N}
\end{array}\right]
$$

Then

$$
\left[\begin{array}{c}
E_{N-1} \\
H_{N-1}
\end{array}\right]=M_{N-1} M_{N}\left[\begin{array}{l}
E_{N} \\
H_{N}
\end{array}\right]
$$

In this way, we have the transmission equation when light passes through all the layers

$$
\left[\begin{array}{l}
E_{1} \\
H_{1}
\end{array}\right]=M_{1} M_{2} \cdots M_{N-1} M_{N}\left[\begin{array}{l}
E_{N} \\
H_{N}
\end{array}\right]=\left[\begin{array}{ll}
m_{11} & m_{12} \\
m_{21} & m_{22}
\end{array}\right]\left[\begin{array}{l}
E_{N} \\
H_{N}
\end{array}\right]
$$

Based on Eq.(4), the reflection coefficient and transmission coefficient of the whole structure can be further drawn:

$$
\begin{aligned}
& r=\frac{\left(m_{11}+m_{12} p_{1}\right) p_{0}-\left(m_{21}+m_{22} p_{1}\right)}{\left(m_{11}+m_{12} p_{1}\right) p_{0}+\left(m_{21}+m_{22} p_{1}\right)} \\
& t=\frac{2 p_{0}}{\left(m_{11}+m_{12} p_{1}\right) p_{0}+\left(m_{21}+m_{22} p_{1}\right)}
\end{aligned}
$$

Where, ${ }^{p}=\sqrt{\varepsilon / \mu} \cos \theta, \varepsilon$ is the dielectric constant, $\mu$ the permeability ratio, $p_{0}$ the external environment coefficient on the left side, $p_{1}$ the external environment coefficient on the right side. Then, we have reflectivity ${ }^{R}=|r|^{2}$, transmittance $T=1-R$. Matlab programming the computing equations, we can simulate the transmission properties of PhCs.

\section{Simulation and design of PDT instrument}

\section{Simulation and design of PhCs filter}

Based on Matlab simulation of band gap, PhCs structure $(A B)_{5} C(B A)_{s}$ is finally determined to meet the design requirement. It is a mingle of three dielectric media, namely CdTe (media A), $S i O=$ (media $\mathrm{B}), \mathrm{Ta}_{2} \mathrm{O}_{5}$ (media C). Media $\mathrm{C}$ is the mingle media, and the media on both sides are of the periods of 5.

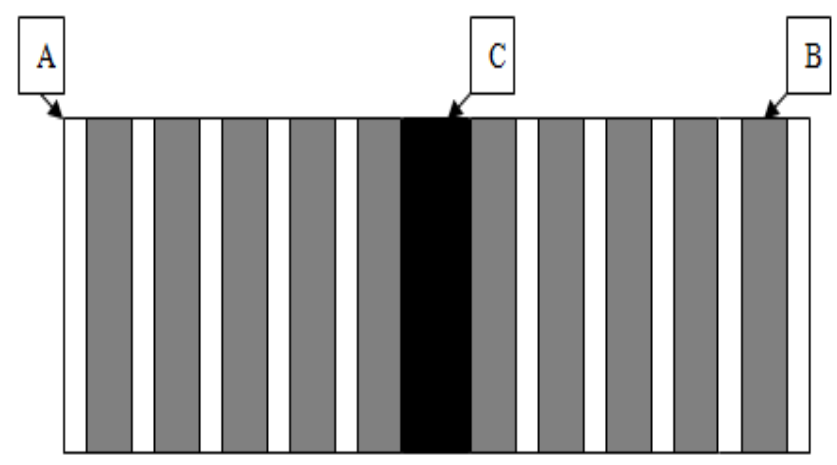

Fig.1 Structure diagram of the photonic crystal designed

As shown in Fig. 1, at the CdTe layer, the reflectivity $n_{a}=2.69$, film thickness $a^{a}=1 /\left(4 n_{a}\right) \lambda_{0}$, namely $91.1 \mathrm{~nm}$. At the $\mathrm{SiO}=$ layer, $n_{b}=1.46, b=1 /\left(4 n_{b}\right) \lambda_{0}$, namely $167.8 \mathrm{~nm}$. At the $\mathrm{Ta}{ }_{2} \mathrm{O}_{5}$ layer, $n_{c}=2.1, c=2 \times 1 /\left(4 n_{c}\right) \lambda_{0}$, namely 233.3nm. Using transmission matrix, Matlab simulation of the transmission spectrum of one-dimensional PhCs membrane design is shown in Fig.2: 


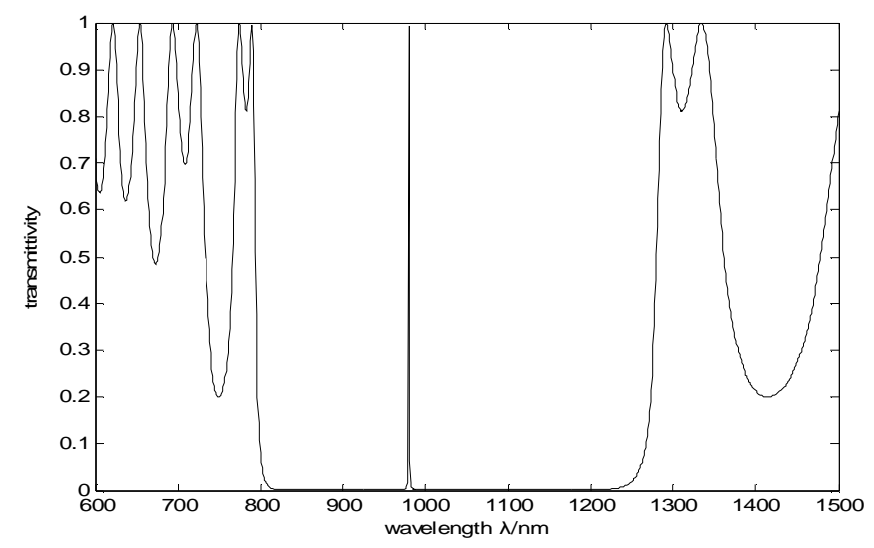

Fig.2 Transmission spectrum of one-dimensional PhCs membrane design

As shown in Fig. 2, the band gap of the one-dimensional PhCs membrane design ranges from 850nm to $1200 \mathrm{~nm}$, covering the entire pumping wavelength used by the present photodynamic therapy instruments. At $980 \mathrm{~nm}$, there is a high transmittance crazing surface, where the bandwidth is $0.4 \mathrm{~nm}$, and the transmittance ratio as high as 0.9914 . Thus, the designed filter can properly meet the color purity requirement in photodynamic cancer treatment.

\section{Structure of PhCs PDT instrument}

In PhCs PDT instrument, near infrared light is taken as source light, and the designed one-dimensional PhCs filter is combined with the laser transmitter of photodynamic therapy instrument by means of optical filming.

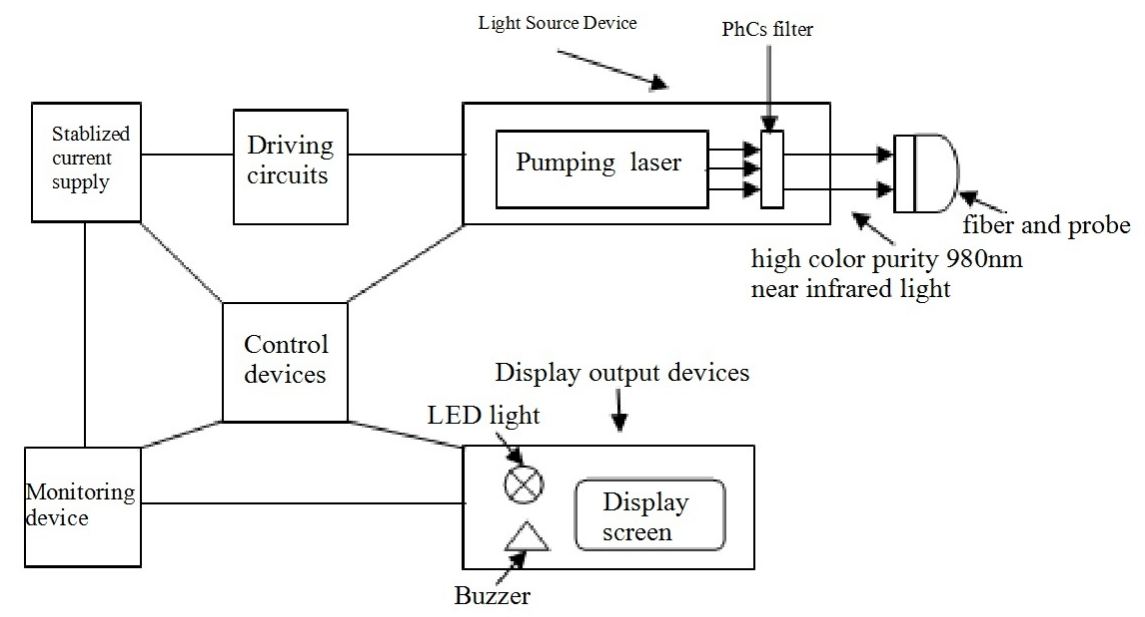

Fig.3 Structure and working principle of PhCs PDT instrument

In Fig. 3, the stabilizing current supply is tunable. It is of high control accuracy, and can provide the dynamics for laser transmission. The pumping laser, as the light source device, emits near infrared light under the driving power of the inner stabilizing current supply and driving circuits. The infrared light emitted is then filtered by the one-dimensional $\mathrm{PhCs}$ structure coated over the pumping laser, ensuring that only the $980 \mathrm{~nm}$ high color purity light can pass through. With the probe connected on the rear, cancer treatment can be conducted over specific parts. The control device controls the switch-on and switch-off of the instrument, monitors the time length of treatment. It can issue an alarm in case of danger. The real-time monitoring device can monitor all modules of the instrument. The device immediately issues an alarm when the value of any parameters exceeds the given standard, and cuts off the power through the control device in case of danger. The display device, composed by a display screen, a red LED light and a buzzer, is used to display time and alarming information. 


\section{Results and Discussion}

In this paper, a method is found to improve the performance of photodynamic therapy instrument based on PhCs technology. The band gap property is applied, the transmission matrix used through Matlab simulation. The one-dimensional mingled PhCs device is of the structure, taking and as periodical structural media, and as mingle media. A PhCs filter is designed which only allows the transmission of the $980 \mathrm{~nm}$ near infrared light. The filter is then coated over the laser transmitter of the photodynamic therapy instrument. In this way, the performance of the instrument is greatly improved, properly solving such technical difficulties as low color purity, unsatisfactory effect of deep treatment. It is simple in structure and stable in performance, and very convenient for the technical improvement of the existing photodynamic instruments. This technology has been granted patent for utility model (PAT. No. ZL201420250994.8) and the application for patent for invention is now under substantive examination (Appl. No. 201410207315.3).

\section{References}

[1] W.M.Star,B.C.Wilson and M.S.Patterson.Implications of photo bleaching for photodynamic therapy[M].New York Photo dynamic therapy,1992.

[2] Reiter,Isabella,Schwamberger,Gtinter,Krammer .Barbara Activation of Macrophage tumoricidal activity by photodynamic treatment in vitro--indirect activation of Macrophages by photodynamically killed tumor cells Journal of Photochemistry and Photobiology B:Biology Volume:50,Issue:2-3,1999:99-107.

[3] Liu H L,Liu F G,Gu Y et al..Several Major Factors Influenced Photodynamic Therapy Effect[J].Chinese Journal of Laser Medicine \& Surgery,2002,11(2):121-124(in Chinese).

[4] Long Z D,Xiong L,Liu Z T et al..The Application of Photodynamic Therapy for Abdominal Malignant Tumors [J].Acta Laser Biology Sinica,2013,3(3),26-28(in Chinese).

[5] E Yablonovitch.Inhibited spontaneous emission in solid-state physics and electronics[J].Phys Rev Lett,1987,58(20):2059-2062.

[6] S John.Strong localization of photons in certain disordered dielectric super lattices[J].Phys Rev Lett, 1987,58(23):2486-2489. 